\title{
The Inverse Infinite Spiral Placement of Prime Numbers and their Derivatives at The Cone of Pythagoras 1:3
}

DOI: https://doi.org/10.24297/jap.v16i1.8437

Vinoo Cameron

Hope Clinic, Athens, Wisconsin, United States

hope9900@frontier.com

\begin{abstract}
All mathematics of placement in this paper is mathematically absolute and precise by clear mathematics. This paper(section1) is the very basic "synopsis" of an upcoming book by the author "The God of Papa Einstein and Sir Isaac Newton ". It delineates the predictable expanding spirals of the prime numbers and their derivatives, by two cords, that hug the half-line of the 1:3 Pythagoras inverse cone. The inner spiral of all predictable spirals of prime numbers and their derivatives and the outer spiral at the predictable template of spiral of prime number $5(+10,+20$ alternate), which is the exact replicate of the inner spiral of prime numbers and their derivatives. The two spirals expand at two separate predictable geometric intervals, the outer spiral expanding predictably slower than the inner spiral as a replicate in geometric placement and in sieve. These prime numbers and their derivatives (Pseudo prime numbers) are additionally sieved by placement in continuum by a predictable formula demonstrated by table at the end of this paper. A postscript at the end of the paper shows the very precise digital phenomena between Prime number 7 and a divisor 5.322/6, a new discovery.

In a separate exhaustive (Section 11) of this paper to be submitted separately, the author along with a contributing physicist discusses the synopsis of the patent configuration of prime numbers at the 1:3 cone , that is the genesis of the curvature of space and form at -1 zero by the placement of prime numbers and their derivatives. "The mother of all science is mathematics", this paper celebrates that dictum.

Method: The resolution of the mathematics is presented in this paper by 4 sections in an unorthodox expression of precise mathematics. The author has chosen to use very simple mathematical language to express complex resolutions to simplify understanding of the complex placement of these numbers and the sieve of prime numbers by continuum.
\end{abstract}

Keywords: Prime number placement, Inverse cone of Pythagoras 1:3

\section{Basic placement of numbers at base 3 of the cone by absolute precise mathematics}

At The 1:3 Cone the numbers naturally place at +3 at the base half -line and +4 at the hypotenuse by the simple mathematical equalization of $+3,+4$ at any base $N$, and upright $n$ at $1: 3$ Pythagoras, $N / n=3$ and $N$ $+n=4$ (upright1). This is shown by diagram as precise +3 at the base and +4 at the hypotenuse.

\section{Diagram 1}

This series comprised of prime numbers and their derivatives is simple and precise by alternate $+2,+4$ and shown by many methods in the text of the book, but for the purpose of this paper, the series is derived by the following patent infinite rhythm of placement of these vital numbers at $+2,+4$ alternating. 
$-1+2=1$

$(1+4) 5+2=7+4=11+2=13,17,19,23,25,29,31,35,41,43,47,49,53,55,59,61,65$ so on.

This series is basically and simply expressed at the base by +3 as follows and the placement is very simple and is clearly infinite by basic mathematics which is self-evident. The prime numbers and pseudo prime numbers are clearly and simply arranged in two cords hugging the half-line. The replicate template of prime number 5 is identified as highlighted yellow and the prime numbers and the pseudo prime numbers are shown in red as alternate placement in two cords on each side of the half-line (hugging the half-line).

\begin{tabular}{|c|c|c|c|c|c|c|c|c|c|c|c|}
\hline 5 & 8 & 11 & 14 & 17 & 20 & 23 & 26 & 29 & 32 & 35 & 38 \\
\hline 4 & 7 & 10 & 13 & 16 & 19 & 22 & 25 & 28 & 31 & 34 & 37 \\
\hline 3 & 6 & 9 & 12 & 15 & 18 & 21 & 24 & 27 & 30 & 33 & 36 \\
\hline 4 & 7 & 10 & 13 & 16 & 19 & 22 & 25 & 28 & 31 & 34 & 37 \\
\hline 5 & 8 & 11 & 14 & 17 & 20 & 23 & 26 & 29 & 32 & 35 & 38 \\
\hline 41 & 44 & 47 & 50 & 53 & 56 & 59 & 62 & 65 & 68 & 71 & 74 \\
\hline 40 & 43 & 46 & 49 & 52 & 55 & 58 & 61 & 64 & 67 & 70 & 73 \\
\hline 39 & 42 & 45 & 48 & 51 & 54 & 57 & 60 & 63 & 66 & 69 & 72 \\
\hline 40 & 43 & 46 & 49 & 52 & 55 & 58 & 61 & 64 & 67 & 70 & 73 \\
\hline 41 & 44 & 47 & 50 & 53 & 56 & 59 & 62 & 65 & 68 & 71 & 74 \\
\hline 77 & 80 & 83 & 86 & 89 & 92 & 95 & 98 & 101 & 104 & 107 & 110 \\
\hline 76 & 79 & 82 & 85 & 88 & 91 & 94 & 97 & 100 & 103 & 106 & 109 \\
\hline 75 & 78 & 81 & 84 & 87 & 90 & 93 & 96 & 99 & 102 & 105 & 108 \\
\hline 76 & 79 & 82 & 85 & 88 & 91 & 94 & 97 & 100 & 103 & 106 & 109 \\
\hline 77 & 80 & 83 & 86 & 89 & 92 & 95 & 98 & 101 & 104 & 107 & 110 \\
\hline & & & & & & & & & & & \\
\hline
\end{tabular}




\begin{tabular}{|c|c|c|c|c|c|c|c|c|c|c|c|}
\hline 113 & 116 & 119 & 122 & 125 & 128 & 131 & 134 & 137 & 140 & 143 & 146 \\
\hline 112 & 115 & 118 & 121 & 124 & 127 & 130 & 133 & 136 & 139 & 142 & 145 \\
\hline 111 & 114 & 117 & 120 & 123 & 126 & 129 & 132 & 135 & 138 & 141 & 144 \\
\hline 112 & 115 & 118 & 121 & 124 & 127 & 130 & 133 & 136 & 139 & 142 & 145 \\
\hline 113 & 116 & 119 & 122 & 125 & 128 & 131 & 134 & 137 & 140 & 143 & 146 \\
\hline & & & & & & & & & & & \\
\hline & & & & & & & & & & & \\
\hline 150 & 153 & 156 & 159 & 162 & 165 & 168 & 171 & 174 & 177 & 180 & 183 \\
\hline 149 & 152 & 155 & 158 & 161 & 164 & 167 & 170 & 173 & 176 & 179 & 182 \\
\hline 148 & 151 & 154 & 157 & 160 & 163 & 166 & 169 & 172 & 175 & 178 & 181 \\
\hline 149 & 152 & 155 & 158 & 161 & 164 & 167 & 170 & 173 & 176 & 179 & 182 \\
\hline 150 & 153 & 156 & 159 & 162 & 165 & 168 & 171 & 174 & 177 & 180 & 183 \\
\hline 186 & 189 & 192 & 195 & & & & & & & & \\
\hline 185 & 188 & 191 & 194 & $\ldots \ldots \ldots$ & So & On & & & & & \\
\hline 184 & 187 & 190 & 193 & & & & & & & & \\
\hline
\end{tabular}

As a Rule of Mathematical Placement at the half line I of 1:3 cone, note the following absolute resolutions of the above table.

a. The template of 5 , the outer replicate spiral, is precisely at $+10,+20$ alternate in two cords as follows precisely the replicate of all spirally placed prime/pseudo prime numbers: 5, 25(5*5),35(5*7), 55(5*11), 65(5*13),85(5*17),95(5*19), 115(5*23), 125(5*25), 145(5*29), 155(5*31), 175(5* $35), 185(5 * 37), 205(5 * 41), 215(5 * 43), 235(5 * 47), 245(5 * 49), 265(5 * 53), 275(5 * 55), 295(5 * 59)$....so on.

b. All prime numbers and their derivatives the pseudo prime numbers of the inner spiral, as a rule of this placement are derived from based numbers 1-19, and also as a rule all these numbers are placed at precise spiral (alternate, at the two cords) by the rhythm $+10,+20$, precisely as follows

Basic numbers constant $1,5,7,11,13,17,19$. The rest of all the prime numbers / pseudo prime numbers in sequence, in the series are derived from these primary numbers and as a rule all are placed at the precise rhythm of $+10,+20$, precisely in order, in spiral placement at the 2 cords at the half-line, as in the above table. 


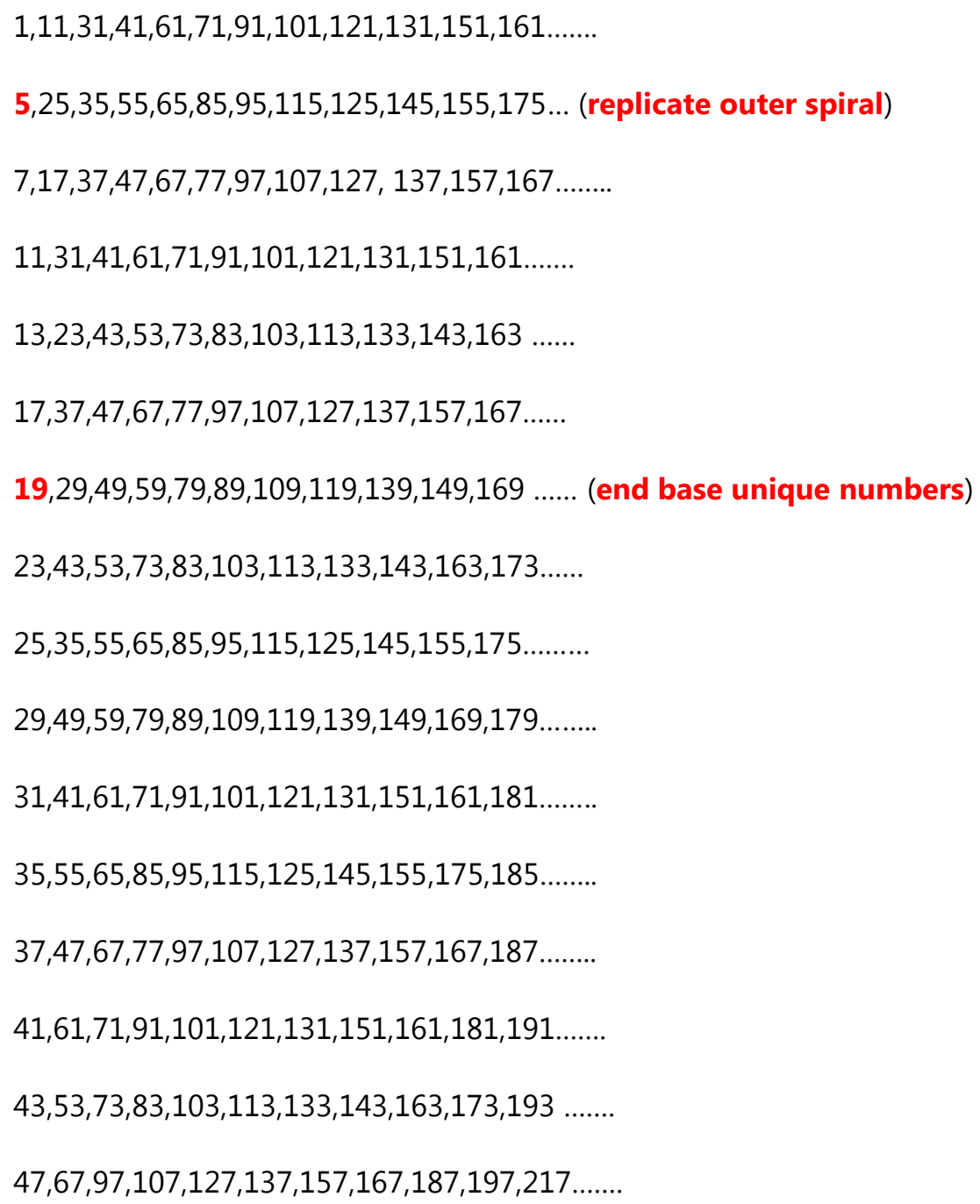

And so on for all prime-numbers and their derivatives in sequence in the series by precise absolute order of placement and gaps.

c. The outer replicate spiral of primer number 5 by template advances over the inner spiral by precise geometric gaps at the table, as a rule by the following precise calculus of geometric expansion (which is expressed mathematically by tangent/curve, as will be discussed in section 11 of the paper, to be submitted separately.)

Physical placement gaps of the spirals at the half line is predictable as the spirals (inner prime and pseudoprime) and an (outer replicate of prime number 5 ) expand predictably at the geometric half line by precise gaps as is seen in the represented table above (factor of 2:6 and $8: 16$, spiral progression) as shown and referenced by the geometric placement and the table above

5 and $25=6(20)$

7 and $35=8(28)$ 
11 and $55=14(44)$

13 and $65=16((52)$

17 and $85=22(68)$

19 and $95=24(76)$

23 and $115=30(92)$

25 and $125=32(100)$

29 and $145=38(116)$

31 and $155=40(124)$

35 and $175=46(140)$ and so on as a rule for all numbers in the series in sequence

\section{Placement of the table of spirals as a precise fit at the Cone at 1:3 Pythagoras.}

In the following precise placement at the $1: 3$ cone the half line is shown in bold and all spiral placements are as in the table and are not highlighted. This representation by Cone placement is precise and infinite in the spiral expansion as described above and fully expressed in the Section11 of this paper to be submitted separately.

$5 . \ldots 4: 3: 4 \ldots .5$

8:7:6: $7: 8$

12:11:10: 9:10:11:12

16:15:14: 13:12:13: 14:15:16

20:19:18: 17:16:15: 16:17:18: 19:20

24:23:22: 21:20:19: 18:19:20: 21:22:23:24

28:27:26: 25:24:23: 22:21:22: 23:24:25: 26:27:28

32:31:30: 29:28:27: 26:25:24: 25:26:27: 28:29:30: 31:32

36:35:34: 33:32:31: 30:29:28: 27:28:29: 30:31:32: 33:34:35:36

40:39:38: 37:36:35: 34:33:32: 31:30:31: 32:33:34: 35:36:37:38:39:40 
3. The geometric placement sieve of all prime numbers from the basic series of Prime numbers/. Pseudo prime numbers as shown above by Continuum and by Numbers formula at 1:3

This calculus is precise discovery of the author, it is as a rule a precise segregation of prime numbers from the pseudo- primer numbers by continuum. It segregates from the main series of Prime numbers and Pseudo prime numbers (discussed above) all the prime numbers from the generated list of exclusive pseudo prime numbers derivatives. This sieve is infinite by a continuum of numbers as placed in the cone spirals. From the extrapolation of this calculus, the exact placement of Prime numbers by geometry at the cone is evident. This requires very careful understanding of the calculus, as it is absolute and precise mathematics calculus at 1:3 cone. There is no error by this one method as shown below, needs basic mathematics as this is precise starting at 7 of the infinite patent series.

At prime number $\mathbf{7 ( 2 1 )}$ : The placement gaps are $\left(6^{*} 7=42\right),\left(7^{*} 12=84\right) \ldots$ Thus the rhythm is 42:84:42:84:42 ... (6, 12 recurrent).

At prime number 11(33): The placement gaps are $\left(6^{*} 11=66\right),\left(12^{\star} 11=132\right)$. Thus, the rhythm is $\mathbf{6 6 : 1 3 2 : 6 6 :}$ 132:66:132 ... $(6,12 ; 6,12$ recurrent).

At prime number 13(39): The placement gaps are $6 * 13=78$ and $12 * 13=156$. Thus, the rhythm is

78:156:78: 156:78:156 ... (6, 12 recurrent).

At 17(51) the placement gaps are $6 * 17=102$ and $12 * 17=204$. Thus the rhythm is 102:204:102:204 (6, 12 recurrent).

At 19(57): the placement gaps are 114:228:114:228, . . (6, 12 recurrent).

AND so on for all numbers in the series in continuum. The following is the precise example for the few numbers at the infinite series as all pseudo prime numbers in red are identified for all numbers in infinite continuum, and the prime numbers never show up in the pseudo prime number calculus

\begin{tabular}{|c|c|c|c|c|}
\hline $\begin{array}{l}{ }^{21(7)} \\
\text { Value/3=x }\end{array}$ & $\begin{array}{l}33(11)(66: 132) \\
\text { Value } / 3=x\end{array}$ & $\begin{array}{l}39(13)(78: 156) \\
\text { Value/3 = x }\end{array}$ & $\begin{array}{l}51(17)(102: 204) \\
\text { Value/3=x }\end{array}$ & $\begin{array}{l}57(19)(114: 228) \\
\text { Value/3=x }\end{array}$ \\
\hline $\begin{array}{c}21+84=105 \\
21+84=105: 15\end{array}$ & $33+132=165 / 11=15$ & $39+156=195 / 13=15$ & $51+204=255 / 17=15$ & $57+228=285 / 19=15$ \\
\hline $\begin{array}{l}105+42=147 \\
: 49\end{array}$ & $\begin{array}{l}165+66=231 . \\
: 77\end{array}$ & $\begin{array}{l}195+78=273 . \\
: 91\end{array}$ & $\begin{array}{l}255+102=357 . \\
: 119\end{array}$ & $\begin{array}{l}285+114=399 . \\
: 133\end{array}$ \\
\hline $\begin{array}{l}147+84=231 \\
: 77 .\end{array}$ & $\begin{array}{l}231+132=363 . \\
: 121\end{array}$ & $\begin{array}{l}273+156=429 . \\
: 143\end{array}$ & $\begin{array}{l}357+204=561 . \\
: 187\end{array}$ & $\begin{array}{l}399+228=627 . \\
: 209\end{array}$ \\
\hline $231+42=273$ & $363+66=429$. & $429+78=507$. & $561+102=663$. & $627+114=741$ \\
\hline
\end{tabular}




\begin{tabular}{|l|l|l|l|l|}
\hline$: 91$ & $: 143$ & $: 169$ & $: 221$ & $: 247$ \\
\hline $273+84=357$ & $429+132=561$. & $507+156=663$. & $663+204=867$. & $741+228=969$. \\
& $: 187$ & $: 221$ & $: 289$ & $: 323$ \\
\hline $357+42=399$ & $561+66=627$. & $663+78=741$. & $867+102=969$. & $969+114=1083$ \\
$: 133$ & $: 209$ & $: 247$ & $: 323$ & $: 361$ \\
\hline $399+84=483$ & $627+132=759$. & $741+156=897$. & $969+204=1173$ & $1083+228=1311$ \\
$: 161$ & $: 253$ & $: 299$ & $: 391$ & $: 437$ \\
\hline $483+42=525$. & $759+66=825$ & $897+78=975$ & $1173+102=1377$ & $1311+114=1425$ \\
$: 175$ & $: 275$ & $: 325$ & $: 459$ & $: 445$ \\
\hline $525+84=609$. & $825+132=957$ & $975+156=1131$ & $1377+204=1581$ & $1425+228=1653$ \\
$: 203$ & $: 319$ & $: 377$ & $: 527$ & $: 551$ \\
\hline $609+42=651$ & $957+66=1023$ & $1131+78=1209$ & $1581+102=1683$ & $1653+114=1767$ \\
$: 217$ & $: 341$ & $: 403$ & $: 561$ & $: 589$ \\
\hline
\end{tabular}

\section{Conclusion and discussion}

If examined and reviewed carefully this very basic mathematics is an absolutely precise fit of the spirals at the cone of 1:3. The Mathematics behind this is very extensive resolution is reserved for Section 11 of the paper and concerns the inherent curvature of the universe space and form dictated exclusively by a configuration of prime numbers at a discovered 1:6 span curve, all very complex mathematics that will be presented as Section 11 by this author and a German Physicist to this very journal which did publish the several initial papers of this author as a testament of pure science by peer review .

PostScript: The discovery of the "digital phenomena at divisor 6/5.322 and Prime number 7", as shown here, was incidental to the representative Physics configuration of prime numbers at the 5, 6 curve constant that will feature in section 11 Physics basis of this paper to be submitted later ( by diagram on the cover page of the book ": The God of Papa Einstein and Sir Isaac Newton". This incidental discovery of this divisor seems to be a precise numbers test for naturally placed numbers verses those that are not rational/ natural to the 1:3 placement. This is entered as follows by very precise mathematics as a caveat to this paper. 


\title{
A Digital Phenomena of Prime numbers at $6 / 5.322$ and Prime number 7 , an unusual research
} discovery by "letter to the editor"

\begin{abstract}
This observation on prime numbers is unique and is a byproduct of research into prime number placements. All prime numbers from 1-10151 without exception tested so far by this method have precisely the same digital outcome at the value 6/5.322, which is derived separately by geometry configuration of prime numbers that has basis in Quantum physics: The authors intent in this "short post script" to the journals that he has submitted a paper on placement of prime numbers by spirals is to note this very unusual phenomena, the significance of which is still under research.
\end{abstract}

Method: $6 / 5.322=1.1273957159 ;$ and 5.322/6 $=0.887$. (Exclusive at these divisors)

$1-0.887=0.113$. Both 887 and 113 are prime numbers.

All prime numbers from 1-10151 were tested and without exception this phenomenon is precisely noted in all prime numbers. Other naturally placed numbers including Pseudo prime numbers also exhibit this phenomena for all "natural numbers" but the author has not reviewed the extent of this phenomena and restricted his observation to all primer numbers as shown by example for sample prime numbers 19, 113 and 10151, and all other prime numbers tested within this range follow the same precise digital outcome as noted. Non- natural pseudo- placed numbers that are not placed in the spiral (example,19*10151=192869), and other such non pseudo prime numbers do not follow this rule.)

\#19.

$19 * 7=133$

$133^{*} 1.1273957159=149.9436302147$

$19 * 0.113=2.147$

\#113

$113 * 7=791$

$791 * 1.1273957159=891.7700112769$

$113 * 0.113=12.769$

\#10151(prime)

$10151 * 7=71057$

$71057 * 1.1273957159=80109.3573847063$

$10151 * 0.113=1147.063$

\#192679 ( a natural pseudo - prime number derived from Prime number $10141 * 19=192679$ ).

$192679 * 7=1348753$ 
$1348753^{\star} 1.1273957159=1520578.35400727$

$192679 * 0.113=21772.727$

\#192869(a non- natural pseudo- prime number, derived from Prime number10151*19=192869). This does not follow the rule

$192869 * 7=1350083$

$1350083 * 1.1273957159=1522077.79030942$

$10151 * 0.113=21794.197$

Results and conclusion of the postscript: This strange precise mathematical observation is a fall out from the authors work on prime number placement and seems to suggest that some derivatives of prime numbers are non- natural and do not place and hence do not follow the digital phenomena. It seems all other natural numbers follow the digital Phenomena, although this complex phenomena needs much more research, but this is far too extensive for the author given the fact that the author is concentrating on pure prime number configuration on the physics of the curvature of the universe space by prime number spirals.

Reference Diagram 5,6 (1:6 Curve constant): This diagram will be fully discussed in section 11 of the paper but it is entered here as the value 6/5.322 as above was directly/incidentally derived from this diagram of the configuration of prime numbers and their derivatives

\section{References for this original research:}

1. VINOO Cameron: The case against mathematical Dogma (Precise mathematical Pi value by finite equation=3.14159292035, pure and applied mathematics journal, vol. no 5,2013, PG 169-173

2. Cameron. V. The first ever precise predictive prime number placement, International journal of applied mathematics research 2(3) (2013)345-351) (journal listed by the American mathematics) society)

3. Cameron. V. The spiral code of prime numbers, International journal of applied Mathematics research 2 (2)(2013)279-292)

4. Cameron. V. The unified Theorem at -1 (Vedic Zero), International Journal of Mathematics research, 2(2) (2013) 221-251.

5. Cameron. V, The disproof and fall of the Riemann's hypothesis by quadratic base: The correct variable distribution of prime numbers by the clear mathematics of the half-line values ("Chan function") of prime numbers, International Journal of Applied Mathematical Research, 2(1) (2013) 103-110.

6. Cameron V, den Otter T. Prime numbers 2012. Jam Sci 2012;8(7):329-334]. (ISSN: 1545-1003), http://www.jofamericanscience.org.

7. Cameron V, Prime number Coordinates and calculus J AmSci, 2012; 8(10):9-10]. (ISSN: 1545-1003). http://www.jofamericanscience.org

8. Cameron: Prime number19, Vedic Zero and the fall of western mathematics by theorem. International journal of applied mathematical research 2(1) (2013)111-115 
9. Cameron: The rational variability of all empty space by prime number: International journal of applied mathematical research, 2(2) (2013)157-174

10. Cameron: The poison pill of current mathematics theory, delivered: International journal of applied mathematical research; 2(3) (2013)387-402)

11. Cameron V: The End calculus of mathematics and Prime numbers: International journal of Applied Mathematics, 2(4) (2013)423-438.

12. Cameron V: Pythagoras1:3, an expression of the finite universe of mathematics: International journal

13. -3 as the created root of all mathematics by numbers, prime number 5 as created template of all prime numbers and pseudo prime numbers: Journal of progressive research in Mathematics (JPRM), Volume14, issue1.September 17, 2018

14. Theorem of 1:3 Pythagoras cone and prime number predictive double spirals: Journal of progressive research in mathematics (JPRM), Volume14, issue 1, September 22, 2018.

15. END. Vinoo Cameron M.D FRCS. September $18^{\text {th }}, 2019$ 